\title{
472145 - CONTEXT SENSITIVE PHYSIOLOGICAL MONITORING IN CHILDREN
}

\author{
J Mark Ansermino, FRCPC ${ }^{1}$, Jeremy Daniels, BASc ${ }^{1}$, Joanne Lim, MAsc ${ }^{1}$, Ping \\ Yang, MASc ${ }^{2}$, Chris Brouse, MASc ${ }^{2}$, Guy Dumont, PhD $^{2}$ \\ 1. Anesthesiology, Pharmacology, and Therapeutics, University of British Columbia, \\ Vancouver, BC, Canada \\ 2. Electrical and Computer Engineering, University of British Columbia, \\ Vancouver, BC, Canada
}

Introduction: The clinical diagnosis of an abnormal physiological state is rarely dependant on the value of a single physiological variable. Anesthesiologists monitoring patients judge the current patient state within the context of previous observations, knowledge about the patient, and clinical experience. An automated mathematical process of detecting changes in trends can be accomplished by summing differences between a value's prediction and observations. We have developed a software tool for performing context sensitive monitoring, and detecting key events in intraoperative physiology.

Methods: Heart rate (HR), end tidal carbon dioxide (EtCO2), exhaled minute ventilation (MVexp), and respiratory rate (RR) were modeled using a dynamic linear growth model, whose noise covariances were estimated by an adaptive Kalman Filter based on a recursive Expectation-Maximization method. Events are detected by adaptive Cumulative Sum (CUSUM) testing $(1,2)$. The events in mean noninvasive blood pressure (mean NIBP) and oxygen saturation (SpO2) are detected using CUSUM testing on a forecasting residual from a predictor based on an Exponentially Weighted Moving Averaging Model (3). The algorithms were implemented in a Java® software environment. Following ethical approval, the tool was evaluated in real time alongside current monitors. A median filter was optimized for each trend to remove artifacts and transients. Tuning parameters were fixed based on previous testing. Events from the stable period of anesthesia were marked by the clinician in real time. Each event detected was graded (artifact (A), clinically insignificant (CI), clinically significant (CS), clinically significant with action taken (CSAT), clinically significant due to intervention (CSI)) using a graphical display. Missed events were noted. The usefulness of each event was rated on a $10 \mathrm{~cm}$ visual analogue scale (VAS).

Results: Fifteen anesthesiologists (38 cases; mean duration 103 (SD 4.25) minutes) completed the evaluation in a variety of pediatric surgical cases. The type of events are shown in Table 1. The mean number of events per case was 22.8 (SD 23.4). The usefulness value ranged from 0 to 9.3 (for an EtC02 CSAT event). Only 6 significant events were reported as being missed by the algorithms.

Discussion: The algorithms detected 13 events per hour of anesthesia, however many were related to a single clinical event (e.g. HR \& BP). Fifty percent of events were rated clinically significant (CS, CSAT or CSI). SpO2 had a low number of events but many artifacts. The CSI events could have been suppressed by information about clinical interventions. 
References: 1. Yang P, et al. IEEE Trans Biomed Eng 2006; 53(11): 2211-2219. 2. Yang $P$ et al. 27th IEEE EMBS Conference; 2005. 3. Yang P et al. 28th IEEE EMBS Conference; 2006.

Clinical Evaluation Results

\begin{tabular}{|c|c|c|c|c|c|c|c|}
\hline & Total Events & Artifact $(\%)$ & $\mathrm{CI}(\%)$ & $\mathrm{CS}(\%)$ & $\operatorname{CSAT}(\%)$ & $\operatorname{CSI}(\%)$ & $\mathrm{NR}(\%)$ \\
\hline ETCO2 & 212 & 5.2 & 24.1 & 19.8 & 5.2 & 27.4 & 18.4 \\
\hline HR & 253 & 6.3 & 36.4 & 24.5 & 9.5 & 9.9 & 13.4 \\
\hline MVexp & 145 & 5.5 & 20.7 & 17.9 & 2.1 & 34.5 & 19.3 \\
\hline NIBP & 124 & 3.2 & 28.2 & 36.3 & 16.1 & 9.7 & 6.5 \\
\hline RR & 86 & 2.3 & 9.3 & 9.3 & 3.5 & 47.7 & 27.9 \\
\hline SPO2 & 48 & 37.5 & 20.8 & 6.3 & 2.1 & 10.4 & 22.9 \\
\hline TOTAL & 868 & 6.8 & 26.0 & 21.4 & 7.1 & 22.1 & 16.6 \\
\hline
\end{tabular}

clinically insignificant (CI), clinically significant (CS), clinically significant with action taken (CSAT), clinically significant due to intervention (CSI), not rated (NR) 